\title{
An Apparatus for High Speed Measurements of Small-Angle X-ray Scattering Profiles with a Linear Position Sensitive Detector
}

\author{
Takeji Hashimoto, Shoji Suehiro, Mitsuhiro Shibayama, \\ Kenji SAIJO, and Hiromichi KAWAI \\ Department of Polymer Chemistry, Faculty of Engineering, Kyoto University, \\ Yoshida, Sakyo-ku Kyoto 606, Japan.
}

(Received November 29, 1980)

\begin{abstract}
An apparatus for high speed measurements of small-angle X-ray scattering (SAXS) is described. This apparatus utilizes a $12 \mathrm{~kW}$ rotating anode X-ray generator, a linear position sensitive proportional counter (multicathode delay line PSPC), and a two-parameter multichannel pulse height analyzer (MCA) with 12 kwords (16 bits/word) memory area available for SAXS intensity data as a function of position (scattering angles) and time slice. The twoparameter MCA is constructed within a microcomputer system, by utilizing its $\mathrm{R} / \mathrm{W}$ memory for data storage, and the memory incrementing and real-time CRT display is implemented by using two direct memory access (DMA) controllers. The cycle time of the access is about $10 \mu \mathrm{s}$. The measuring time for SAXS profiles with this apparatus can be shortened approximately by three orders of magnitude in comparison with the measuring time with SAXS apparatuses utilizing a conventional step-scanning goniometer and a conventional X-ray tube, thus permitting time-resolved analyses of SAXS profiles. Some applications of the apparatus to dynamic SAXS measurements are presented for polymeric systems, the preliminary results of which seem to indicate the possibility of obtaining a new class of data on dynamics in structural transformation, deformation, formation and annihilation in the scale of a few tens to several hundred Angstroms.
\end{abstract}

KEY WORDS Small-Angle X-ray Scattering / Position Sensitive Proportional Counter / Rotating Anode X-ray Generator / Two-Parameter Multichannel Pulse Height Analyzer / Dynamic SAXS / Block Polymer / Phase Separation / Ionic Cluster / Dynamics of Deformation / Oriented Crystallization /

Small-angle X-ray scattering (SAXS) technique has recently achieved a great advance owing to developments of a position sensitive X-ray detector (PSD), ${ }^{1-10}$ high flux $\mathrm{X}$-ray sources, (such as sources from $\mathrm{SOR}^{11-14}$ and a high-power rotating anode generator), and electronics associated with the detector. The PSD and high flux X-ray source greatly shorten the measuring time of SAXS profiles, thus permitting dynamic studies of structure formation during crystallization, ${ }^{15 a}$ phase-transformation of crystalline polymers ${ }^{15 \mathrm{~b}}$ and phase-separation in polymer blends and block polymers or of their structure annihilations. ${ }^{16,17}$ The dynamic SAXS technique, of course, can be applied to the study of the dynamics of polymer deformation. In this paper, we describe an apparatus constructed for the high speed measurement of SAXS profiles and various applications of this technique to the study of the time dependence of structure change in polymeric systems.

\section{APPARATUS}

We shall first describe an outline of the apparatus (section 1), and this will be followed by detailed descriptions of the optical system (section 2), microcomputer MCA (section 3) and software (section 4), and some performances on the PSD and associated electronics (section 5).

\section{Outline of the Apparatus}

Figure 1 shows an outline of the optical system of 


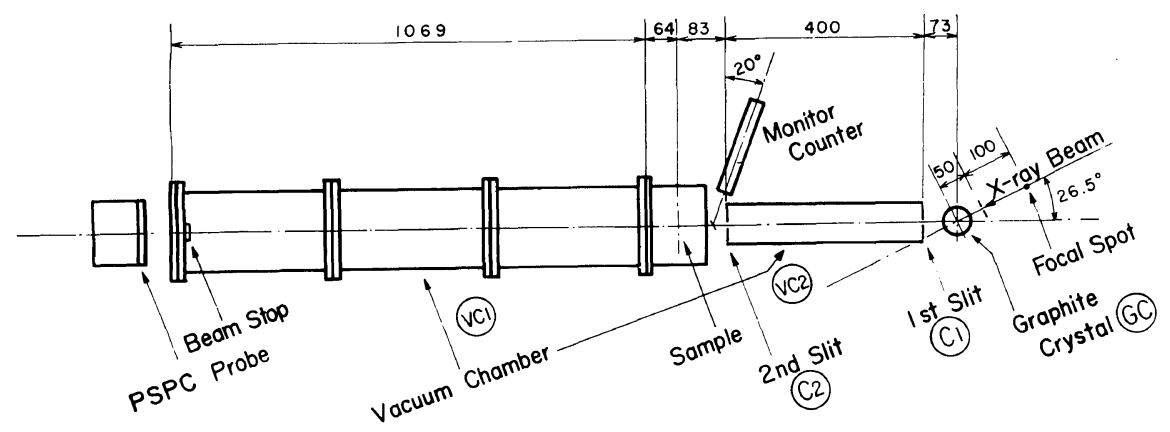

Figure 1. A schematic diagram of the optical system of SAXS apparatus with a linear PSPC: GC, a graphite monochromator; $\mathrm{Cl}$ and $\mathrm{C} 2$, collimating pinholes or slits mounted on $x y$ stages; $\mathrm{VCl}$ and $\mathrm{VC} 2$, vacuum path; PSPC, a linear position sensitive proportional counter.

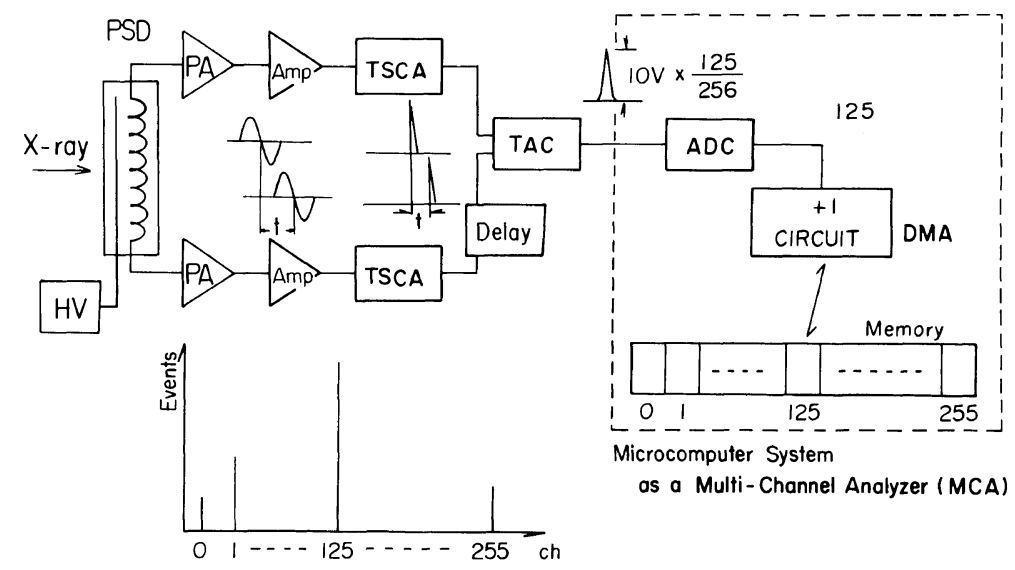

Figure 2. A schematic diagram showing the principle of scattering profiles detection in the PSD system.

the SAXS apparatus with the PSD. We use a $12 \mathrm{~kW}$ rotating anode X-ray generator (RU-a, Rigaku Denki) for a high speed detection of SAXS profiles. We monochromatized the $\mathrm{Cu}-K \alpha$ beam by a graphite crystal (GC) and collimated the beam by a set of pinholes or slits ( $\mathrm{Cl}$ and $\mathrm{C} 2)$. The camera distance between the specimen and the PSD is varied in three steps; 480,830 , and $1180 \mathrm{~mm}$ by connecting as many beam tubes as desired (from 1 to 3) for a vacuum path (VC1). The scattered X-ray intensity distribution is detected simultaneously over many scattering angles by the linear PSD. The fluctuation in incident beam intensity was monitored by counting scattered photons from a thin aluminum foil ( $10 \mu \mathrm{m}$ thick) placed in the path of incident beam with a proportional counter. The sample holder, the monitor counter, the vacuum paths $\mathrm{VCl}$ and $\mathrm{VC} 2$ and the detector are all mounted on an optical bench provided with adjusting devices for level, hight, and an alignment of its center line with respect to the glancing angle of the graphite monochromator.

Figure 2 schematically illustrates the principle of the PSD system. The linear PSD used in this work is a multicathode delay line type proportional counter (Rigaku Denki, manual no. MJ 510 EC). An electric pulse induced on the multicathode by an incident photon is transmitted through the delay line to both ends of the detector. The pulses at the both ends of the delay line are fed into the amplifiers (AMP) where the pulses are doubly differentiated. The bipolar output signals are fed into the timing single channel analyzers (TSCA). The zero cross detectors (ZC) in the timing single channel analyzers (TSCA) give trigger pulses at time when the outputs of the AMP cross zero. According to the position of incident photon, the two pulses of $\mathrm{ZC}$ have a certain time lag. This time lag $t$ is then 
converted into a voltage signal by a time-toamplitude converter (TAC). The TSCA has the function of a pulse-height analyzer which may be used for eliminating the higher-order harmonics of Bremstrahlung radiation from the graphite monochromator.

The full delay of our detector was $800 \mathrm{~ns}$ and was converted to a voltage signal of $10 \mathrm{~V}$. The voltage signal from 0 to $10 \mathrm{~V}$ is then converted to a digital number by an analogue-to-digital converter (ADC), maximum conversion gain and maximum conversion time being $1024 / 10 \mathrm{~V}$ and $10 \mu \mathrm{s}$, respectively. The conversion gain is variable, Figure 2 corresponding to $256 / 10 \mathrm{~V}$ ( 8 bit resolution), i.e., thus the effective length of the PSD being resolved into 256 channels (chs.). The hardware memory incrementer adds +1 (increment mode) or -1 (decrement mode) to the content of read/write $(\mathrm{R} / \mathrm{W})$ memory of microcomputer, (the address of which is specified by the ADC output) by using a direct memory access (DMA) technique, the memoryincrementing cycle being $10 \mu \mathrm{s}$. All of the functions of this built-in multichannel pulse-height analyzer (MCA) can be controlled by the microprocessor, which enabled us to carry out a wide variety. of experiments including time-resolved studies.

The information stored in $\mathrm{R} / \mathrm{W}$ memory can be displayed on an oscilloscope during the data acquisition (by using the DMA technique as will be described in section 3). The stored information in $\mathrm{R} / \mathrm{W}$ memory can also be displayed by a chart recorder and a printer through PIAs and DACs, transferred into digital cassette tapes, and sent to the University Computer Center through an acoustic coupler for further analyses of data, all of which are carried out by software as will be discussed later in Figure 8. The control of the system is performed through a console typewriter (Silent 700, Texas Instrument) (see Figure 8). The interval timer can be used to specify measuring time (PT), time interval between successive measurements (TI), and duration of the entire experiment which can be controlled over a period from $10 \mathrm{~s}$ to a few days.

An elaborate software system has been developed so that the microcomputer MCA can be easily controlled by means of a BASIC-type high level language.

\section{Optical System}

In this section we shall describe details of the

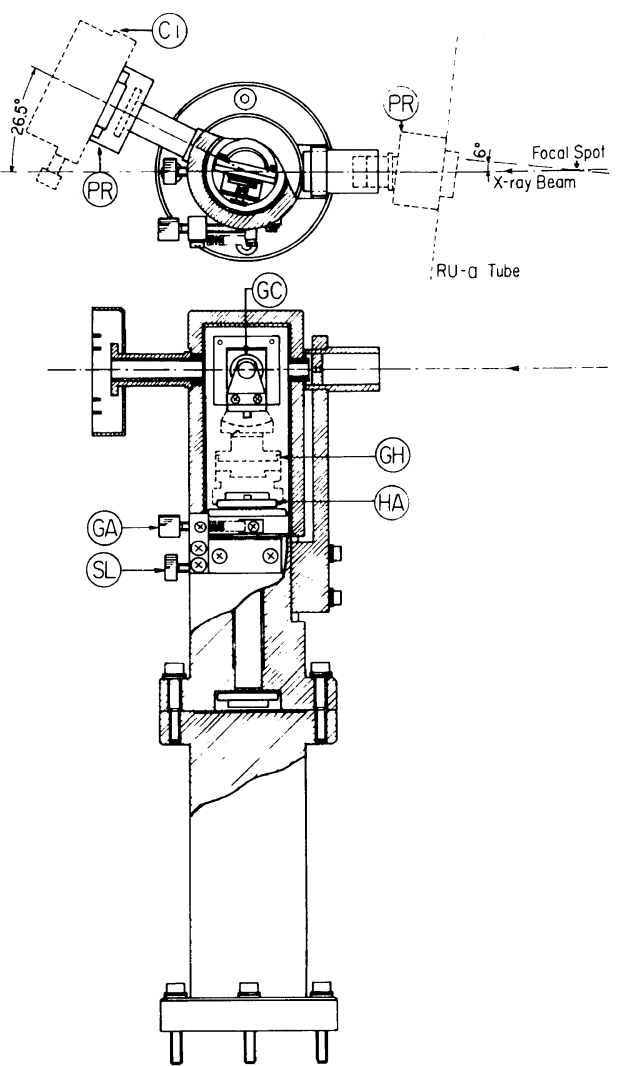

Figure 3. A holder for a graphite monochromator: $\mathrm{GC}$, a flat piece of pyrolytic graphite of $25.4 \mathrm{~mm}$ square; $\mathrm{GH}$, a goniometer head for holding a single crystal; GA, a micrometer for adjusting a glancing angle; $\mathrm{SL}$, a lock of $\mathrm{GA} ; \mathrm{Cl}$, a collimator; $\mathrm{HA}$, a fine adjustment for height of monochromator.

optical system of the apparatus.

Figure 3 shows, the holder for the graphite monochromator and attachments for adjusting the monochromator with respect to the center line of the incident X-ray beam. The entire monochromator holder is mounted on a surface plate of the X-ray generator. A small, flat piece of pyrolytic graphite of $25.4 \mathrm{~mm}$ square (GC) is mounted on a goniometer head $(\mathrm{GH})$, originally constructed to hold a single crystal in the four-circle goniometer. The head has adjustable devices for shifts along the $x$ and $y$ axes $( \pm 3 \mathrm{~mm}$ ) (in a plane perpendicular to the propagation direction of incident beam which is taken in the $z$-direction) and for the tiltings along the two axes $\left( \pm 20^{\circ}\right)$. The $x$ and $y$ axes are taken in horizontal and vertical directions, respectively. The 

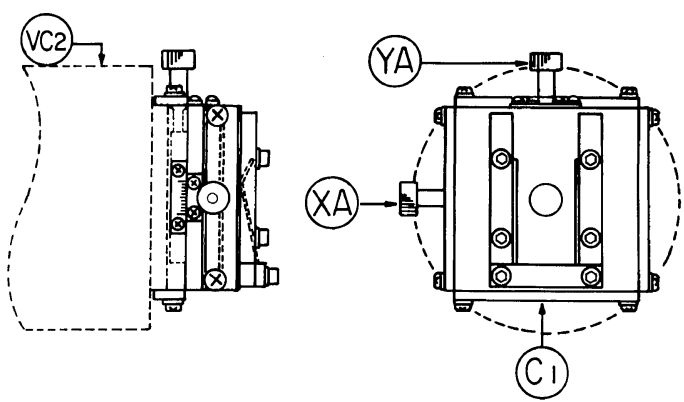

Figure 4. The collimator $\mathrm{Cl}: \mathrm{XA}$ and $\mathrm{YA}$, screws to adjust the pinhole or slit along $x(=5 \mathrm{~mm})$ and $y(=\mathrm{mm})$ directions, respectively.

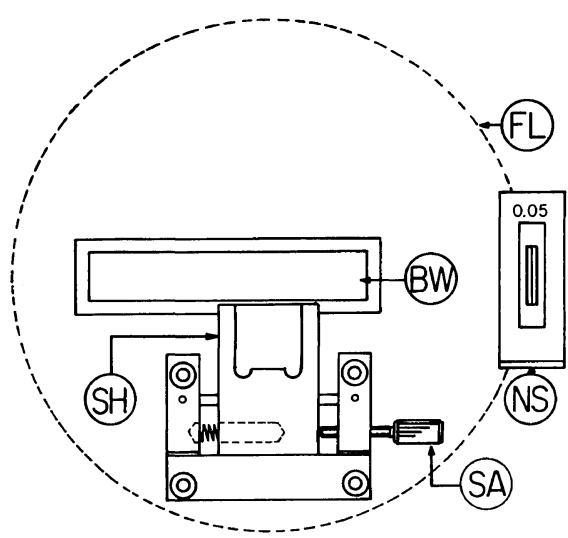

Figure 5. A holder (SH) for a fine slit of $50 \mu \mathrm{m}$ (NS) is mounted on one side of the flange FL (i.e., outside of the vacuum path). The slit is used to measure position resolution, linearity between the position and channel number, the resolution reduction of the detector due to oblique incidence of photons, and the detector sensitivity along its length.

glancing angle of the goniometer is adjusted to $26.5^{\circ}$ by a micrometer (GA) and is locked by a screw (SL). The parts designated as PR is a protector which prevents $\mathrm{X}$-ray radiation leakage. The $\mathrm{C} 1$ part is a collimator and has a pinhole or slit holder mounted on $x y$ stage. A fine adjustment for the height of monochromator with respect to the incident beam is carried out by a screw (HA).

Figure 4 shows the part of the collimator $(\mathrm{Cl}$ or $\mathrm{C} 2$ in Figure 1), which has a slit or a pinhole holder mounted on $x y$ stage; $\mathrm{XA}$ and YA are screws for adjusting the positions along $x( \pm 5 \mathrm{~mm})$ and $y( \pm 5 \mathrm{~mm})$ axes, respectively, (these positions can be read with an accuracy of $1 / 100 \mathrm{~mm}$ ). The pinholes 0.5 and $1.0 \mathrm{~mm}$ in diameter or the slits of $0.2 \times 3,0.5 \times 3$, and $1.0 \times 3 \mathrm{~mm}^{2}$ can be inserted into the collimators $\mathrm{C} 1$ and $\mathrm{C} 2$ so as to collimate the incident beam, (the slits or pinholes being fixed by leaf springs). Entrance and exit windows of the vacuum path $\mathrm{VC} 2$ are sealed by thin mylar films of $12 \mu \mathrm{m}$ thick.

The entrance window of the vacuum path (VC1) is $26 \mathrm{~mm}$ in diameter and is sealed by a thin mylar film of $12 \mu \mathrm{m}$. The other end of the vacuum path $\mathrm{VCl}$ is a flange (FL) with a window of beryllium (BW) $15 \times 85 \mathrm{~mm}^{2}$ and $0.5 \mathrm{~mm}$ thick as shown in Figure 5, viewed against the propagation direction of the incident beam. On one side of the flange, i.e., the outside the vacuum path, the slit holder $(\mathrm{SH})$ is mounted as shown in Figure 5. On the other side of the flange shown in Figure 5, i.e., inside the vacuum path, an incident-beam stopper is mounted, so that the incident beam is stopped before hitting the beryllium window; this minimizes the parasitic scattering from the window. The position of the directbeam stopper is adjusted by a small synchronous motor.

A narrow slit of $50 \mu \mathrm{m}$ width (NS) can be inserted into the holder ( $\mathrm{SH}$ ) and fixed by a leaf spring; its center is adjusted with respect to the center of the incident beam by a screw (SA). The narrow slit is used for measuring (i) the position resolution of the detector, (ii) the resolution reduction of the detector due to oblique incidence of photons, and (iii) the relationship between the channel number and the position of the photons. For these measurements, a broad center beam or a broad scattered beam profile is used, and a slit (NS) of $50 \mu \mathrm{m}$ width is inserted; its center is adjusted at an appropriate position within the scattered beam or center beam profile. The beam profile used is broad enough so that the beam intensity is essentially constant over the $50 \mu \mathrm{m}$ slit width. The profile of the beam through the slit is measured by the PSD as a function of position of the PSD. That is, the PSD is shifted along the $x$-direction (i.e., horizontally) relative to the slit for measuring (i) and (iii). The profile is also measured as a function of the tilting angle. That is, the PSD is tilted with respect to the $z$ direction so as to measure (ii) the resolution reduction of the detector due to oblique incidence. The results are described in detail elsewhere. ${ }^{18}$

The sample-to-detector distance is varied in three steps $(480,830$ and $1180 \mathrm{~mm})$ by removing one or 
A High Speed SAXS Apparatus with PSD

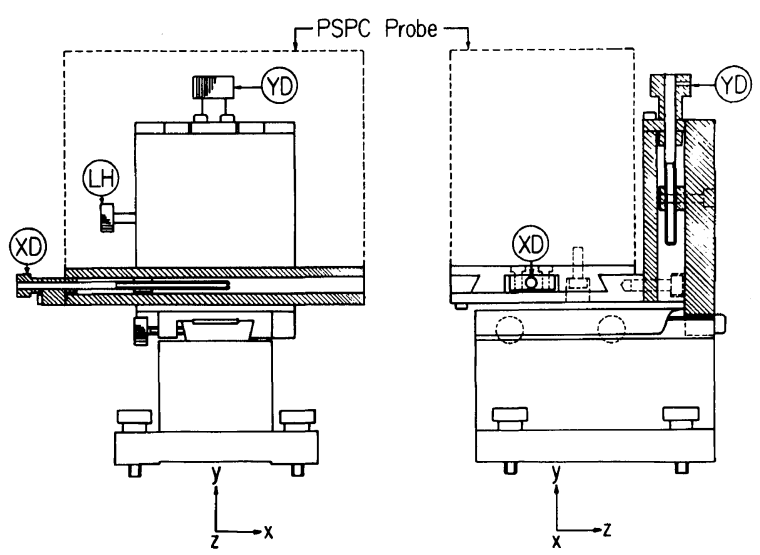

Figure 6. A holder for the PSD: XD and YD, micrometers to adjust the center of the detector along the $x$-axis $(+40 \sim-10 \mathrm{~mm})$ and $y$-axis $(+17 \sim-8 \mathrm{~mm})$ to facilitate making measurements of the slitweighting function and detector performances; LH, a screw lock for height adjustment.
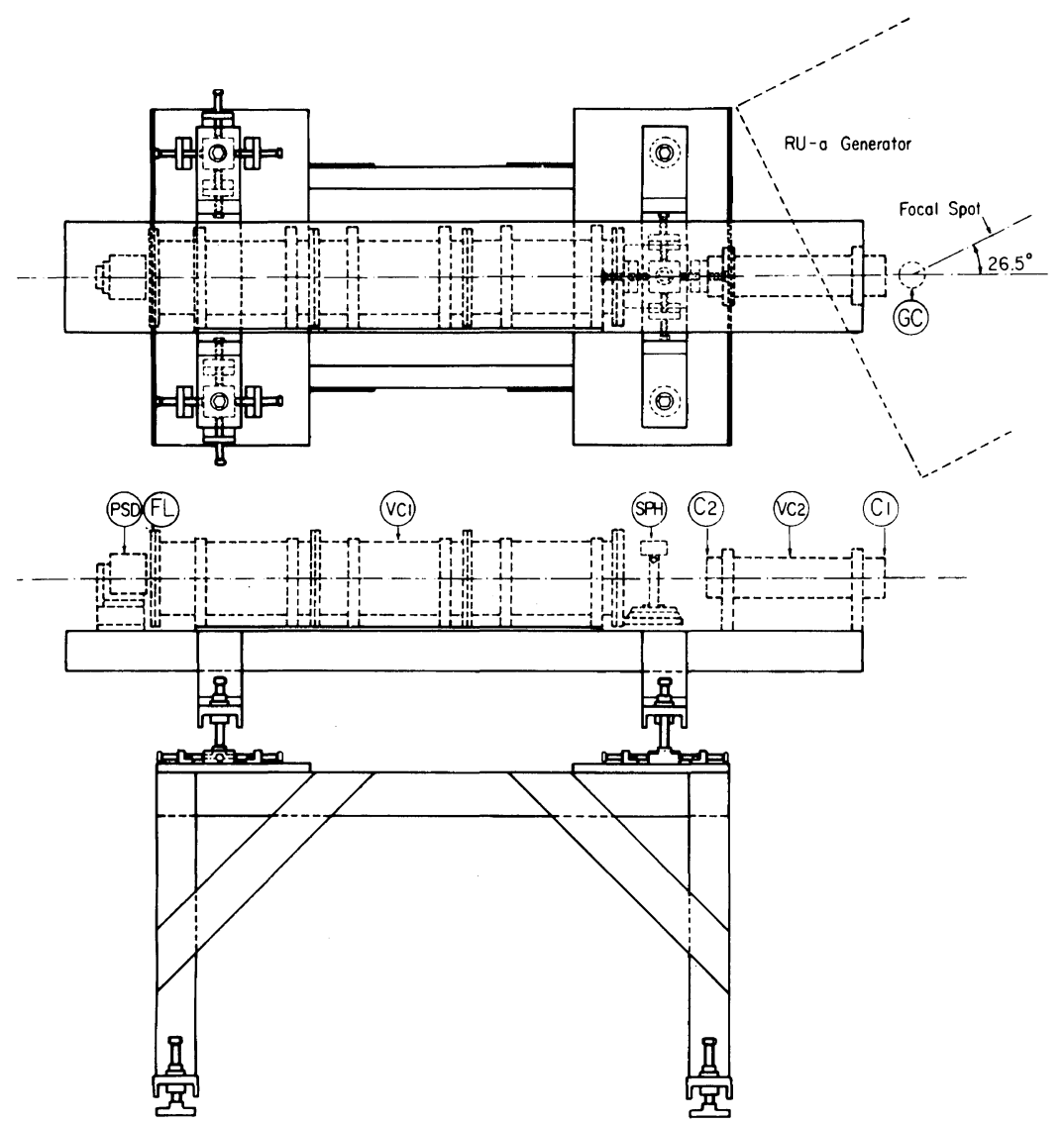

Figure 7. An optical bench on which the collimating optics ( $\mathrm{C} 1, \mathrm{C} 2$, and $\mathrm{VC} 2)$, sample holder (SPH), the vacuum path (VC1) with a beam stopper, and the PSD are mounted. 
two beam tubes $156 \mathrm{~mm}$ in diameter and $350 \mathrm{~mm}$ long. The height of the beryllium window (BW) $(15 \mathrm{~mm})$ is such that even at the greatest camera distance the beryllium window does not act as a slit edge.

The PSD has a beryllium window $10 \mathrm{~mm}$ in height and $58 \mathrm{~mm}$ long. In front of the window, a height limiting slit can be mounted in order to vary the slit-height weighting function. The slits with height $1.0,2.0,4.0$, and $8.0 \mathrm{~mm}$, and $60 \mathrm{~mm}$ long are used. The effective depth of the PSD along $z$ direction is $10.5 \mathrm{~mm}$.

Figure 6 shows a holder for the PSD. The detector holder is designed to facilitate measurement of the slit weighting function of the SAXS system as well as those of the detector performances, such as position resolution, linearity in the relationship between the channel number and position, and resolution reduction due to the oblique incidence of the photons. In order to measure the detector performances and to cover scattering angles as large as possible, the center of the detector is designed to be able to move along the $x$-axis by $+40 \mathrm{~mm}$ and $-10 \mathrm{~mm}$ with a micrometer screw (XD); the displacement can be read with a precision of $1 / 100 \mathrm{~mm}$. In order to measure the weighting function along the slit height, i.e., along the $y$-axis, the center of the detector is designed to be able to move +17 and $-8 \mathrm{~mm}$ along the $y$-axis by a screw (YD) and locked by a screw (LH); the displacement can be read with a precision of $1 / 10 \mathrm{~mm}$. The flange (FL) with a beryllium window is designed so that it can be removed from the beam tube when the slitweighting function is measured. The holder can be moved along the $z$-direction (i.e., along the beam direction) by $20 \mathrm{~mm}$.

Figure 7 shows the design of the optical bench on which the collimating optics ( $\mathrm{C} 1, \mathrm{C} 2$ and $\mathrm{VC} 2)$, the sample holder (SPH), the beam tubes (VC1), and the PSD are mounted. The bench is designed to be stable for mechanical vibration and is also designed to function as a kind of goniometer so that the level and height of the bench and the alignment of center of the bench with respect to the center of incident Xray beam can be adjusted without great difficulty.

\section{Microcomputer MCA}

Considering a high rate of data acquisition regarding the position sensitive $\mathrm{X}$-ray detecting system and the requirement to control complicated experimental conditions in order to meet the needs of a wide variety of the experiments, such as timeresolved experiments, an on-line computer must be used to control data acquisition by MCA and data transfer from MCA to a computer for data analyses. Hashizume and coworkers ${ }^{3}$ used a minicomputer MCA in which the position information from the $\mathrm{ADC}$ is transferred to the minicomputer and memory mapping and incrementing are performed by software. Hendricks ${ }^{9}$ utilized a minicomputer in which an MCA function is implemented by a high speed microprocessor as a memory mapper and by using the custom instruction of the minicomputer as a memory incrementer of $\mathrm{R} / \mathrm{W}$ memory in the computer. Recently, Hendricks and coworkers ${ }^{39}$ have developed a fast digital data acquisition system utilizing a huge four-port bulk memory. An incrementer connected to one of the ports is linked with daisy-chained CAMAC modules to perform memory mapping and time correlation for various kinds of multiparameter experiments. The system is believed to be one of the most powerful and flexible data acquisition systems for position-sensivive Xray and neutron detection with two-dimensional detectors.

However, for a relatively small system (small in terms of memory capacity) such as ours with a linear position detector, a microcomputer can be used as an MCA. Needless to say, general purpose microcomputers are too slow to perform memory mapping and incrementing by software. Consequently a hardware memory mapper and incrementer as an MCA controller are necessary. A part of the $\mathrm{R} / \mathrm{W}$ memory of the microcomputer system is used for data storage. The access to the $\mathrm{R} / \mathrm{W}$ memory of microcomputer by the hardware memory mapper and incrementer is provided by means of a direct memory access (DMA) technique (see Figure 8). We have developed such a microcomputer-MCA system which has proved to be highly efficient and economical. It was possible to build all necessary MCA hardwares other than the data memory on two printed circuit-boards $225 \mathrm{~mm} \times 300 \mathrm{~mm}$, in size.

Figure 8 shows a schematic diagram of our microcomputer MCA system. Information on photons incident upon the PSD is stored in the R/W memory in terms of the number of events at the memory address corresponding to the position of the incident photons. This process is carried out by 


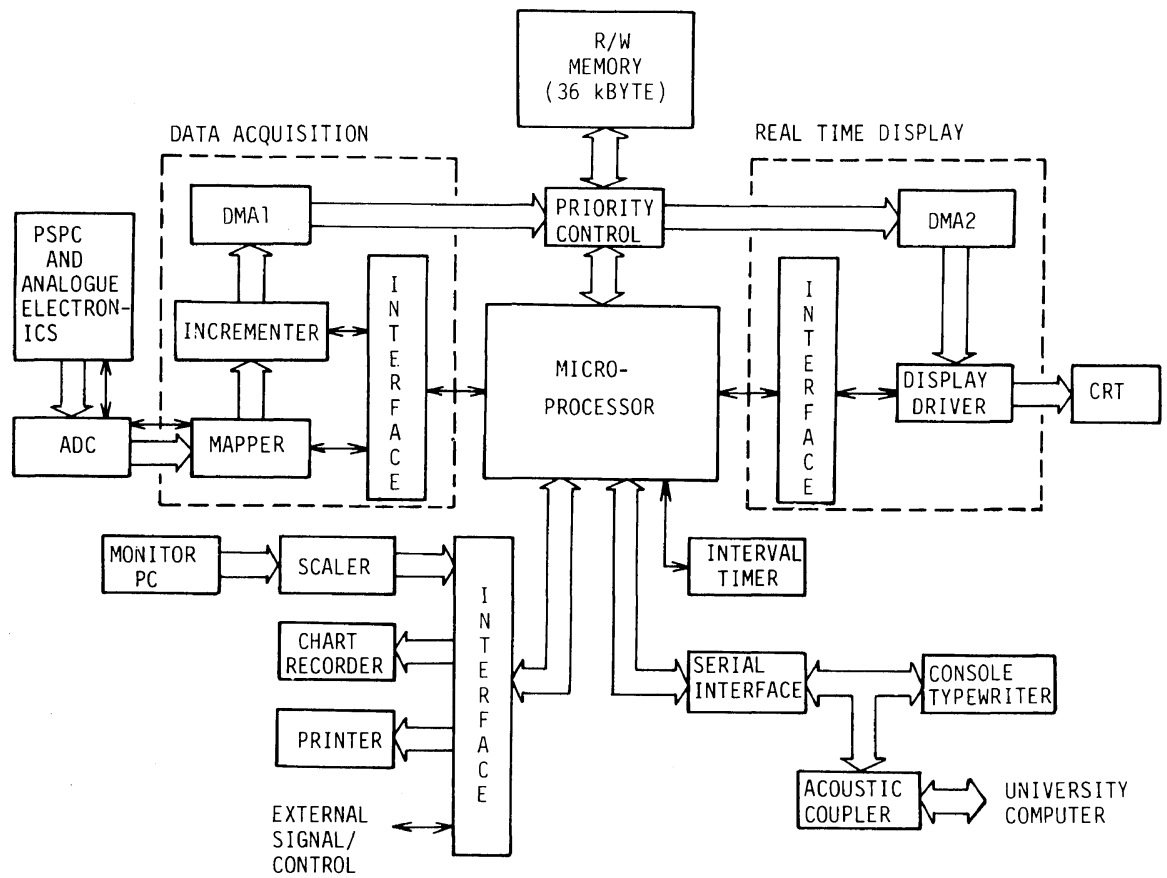

Figure 8. A schematic diagram of the microcomputer-MCA system.

a hardware mapper and incrementer. The memory access by the incremeter is performed under the control of a DMA controller (DMA1). A microcomputer program can be simultaneously and independently executed, while the data acquisition is running. The information stored in the $\mathrm{R} / \mathrm{W}$ memory can be read out by an another DMA controller (DMA2) and displayed on an oscilloscope during the data acquisition through a display driver which has three digital-to-analogue converters (DAC). The DMA1 has the highest priority, the DMA2 the next, and the microprocessor, i.e., the software, the lowest priority for minimizing the dead time arising from the data acquisition. The dead time of the memory incrementing circle is $10 \mu \mathrm{s}$ using this technique. The thin and thick arrows in the diagram denote the control signal and data flows, respectively.

The microcomputer used in this system is a general purpose 8-bit microprocessor-(Motorola M6800)-based system which has 36 kbyte R/W memory. The incrementer and the two DMA controllers handle 16 bit wide word data so that 65535 events can be recorded per channel before overflow occurs.
The mapper, incrementer, display driver, and two DMA controllers are interfaced with the microprocessor through peripheral interface adapters (PIA) and can be controlled by software. Control of the MCA functions are executed by writing various parameters into PIAs connected to an individual hardware which performs the desired functions.

The fluctuation of incident beam power is monitored by a proportional counter as shown in Figure 1. Concurrently with the data acquisition by the MCA, the monitor intensity is counted by a scaler and is followed by transferring its content to a particular memory location by software.

Up to 12 kword ( 24 kbyte) out of 36 kbyte R/W memory can be available for a data area and this enables one to measure the SAXS profiles with 256 channels at 48 time slices, as an example.

Figure 9 shows a schematic diagram of the mapper and associated control logic. Information on a position of incident photons coming from the ADC is concatenated with the content of 12 bit Y-register which is designed to determine the starting address of the currently activated one dimensional data array. The starting address corresponds to that for a particular time slice ( $\mathrm{Y}$-channel) in time-resolved $\mathrm{X}$ - 


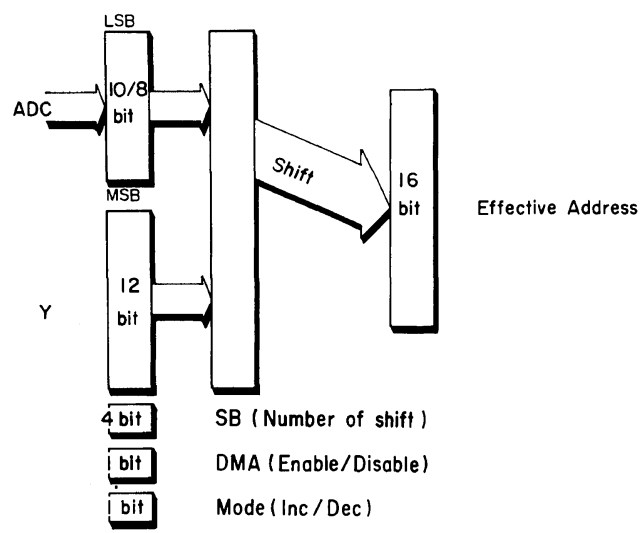

Figure 9. A schematic diagram of the memory mapper, and associated control logic.

ray experiments. The concatenated content of $22 \mathrm{bit}$ shift register is then shifted $s$ places to give an effective address, where $s$ is the content of 4 bit SBregister. The content of the memory in the effective address must be incremented or decremented, according to the content of INC/DEC status bit whenever the DMA enable bit is on. The shift operation described above serves to change the position resolution of the PSD, i.e., the number of $\mathrm{X}$-channels (the number of scattering angles at which photon countings are conducted) and also the time-resolution, i.e., the number of Y-channels. The content of $\mathrm{Y}$ - and SB-registers as well as the other two status control bits are written by software.

When the memory content of a particular effective address reaches 65535 events, the memory incrementing at that address can be either stopped or continued, the selection of the two modes being finished with a jumper on the MCA board. In one of the modes, i.e., a "stop-mode," the memory incrementing is still continued for other addresses whose memory content has not yet overflowed, but is stopped for the addresses whose memory content is overflowed.

\section{Software}

A cassette tape operating system (CTOS) has been developed to operate the microcomputerMCA system. The CTOS has approximately 20 system commands beginning with a slash, "/", followed by the name of a command and/or the name of a subcommand and then by the names of parameters, if necessary.
As one of the system commands, "/EXP" has various subcommands for controlling the MCA functions, such as the starting and stopping of the data acquisition, clearing the data area, activating CRT display, to calculating the logarithmic transformation of a scattering profile, and the recording of one-dimensional data (intensity $v s$. channels) on a strip chart recorder. In addition, the MCA control software has 9 MCA parameters: PT, the preset time, i.e., the duration of photon counting; TI, the time interval between successive dataacquisitions; $\mathrm{XC}$, the number of position points $(\mathrm{X}$ channels); YC, the number of time slices ( $\mathrm{Y}$ channels); IA, the base address of the data acquisition array, FS, the full scale of the vertical axis of the CRT display and so on. There is a special command for printing all MCA parameters so that the user can check the status of the MCA whenever necessary. Furthermore, the command, "/EXP," allows the user to modity any of these parameters simply by specifying the name of each parameter to be modified and the corresponding new value coupled with the sign $(=)$ following a subcommand (e.g., /EXP DISPALY YC $=4 \mathrm{FS}=6$ ).

Other system commands are used to control the microcomputer system in genaral. These are the commands to load, save, and edit programs, manage cassette tape files, and develop assembler language programs.

A high level language $(\mathrm{VTL} / \mathrm{K})$ has been developed for this system..$^{40}$ The $\mathrm{VTL} / \mathrm{K}$ is an easy-touse BASIC like language based on VTL $/ 2^{41}$ and has two byte wide (16 bit) data and 16 bit integer arithmetic which are compatible with the MCA.

The system commands can be placed in a program written in VTL/K and be executed under the control of the program (program mode), or be executed directly by typing the commands (direct execution mode). These two modes gives great flexibility in carrying out experiments. An experiment can be carried out either by typing several commands in an appropriate order, e.g., clear data area, start data acquisition and so on, even in a time-slicing experiment, or by writing and executing a program consisting of a series of system commands.

The program mode provides a means for controlling special kinds of experiments such as deformation studies which must be started in synchronization with an external signal. In addition, we can 
A High Speed SAXS Apparatus with PSD

Table I. Example of a VTL/K program along with results of the execution.

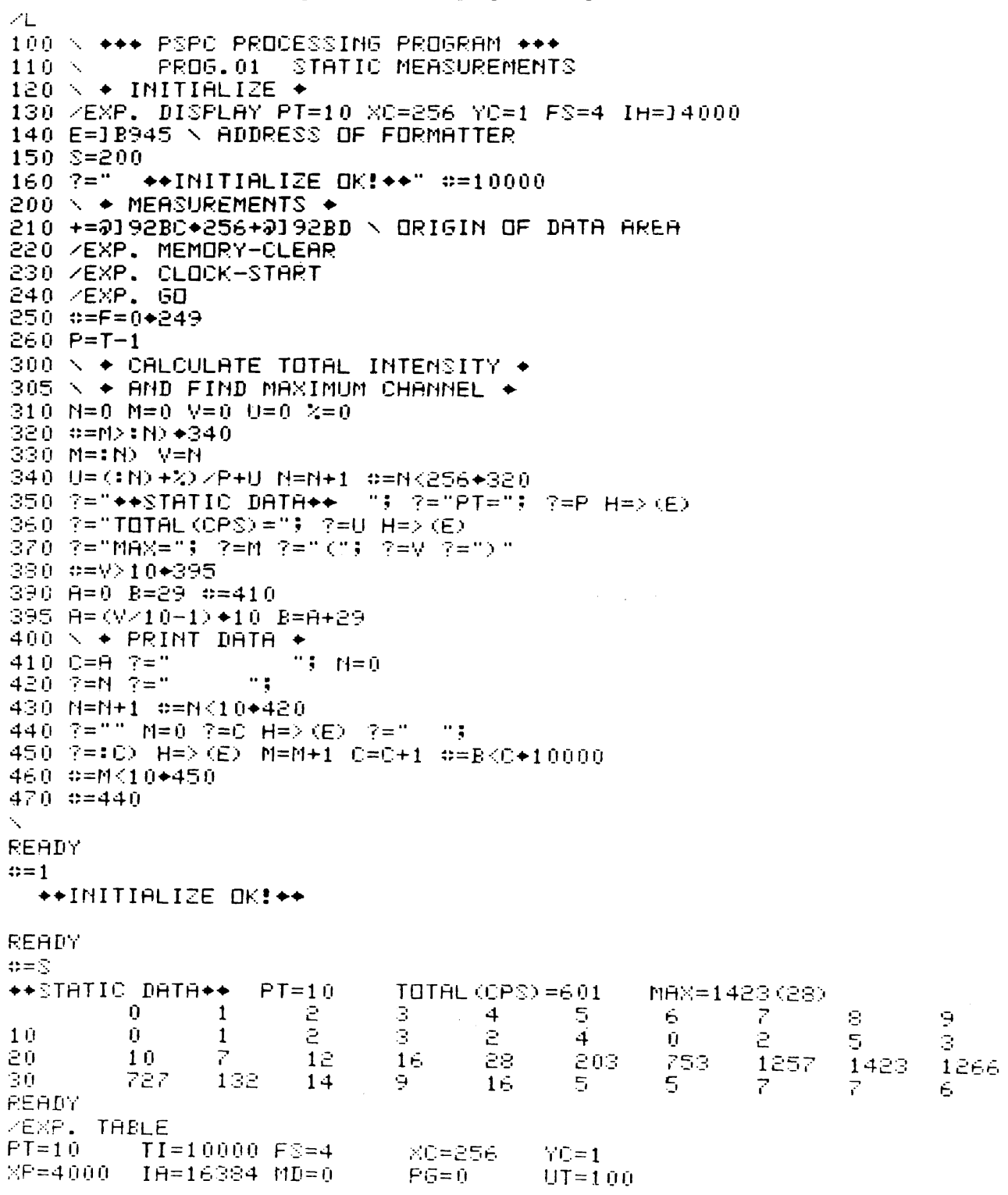

write several programs in order to make some calculations on measured data, to print out a desired portion of data, and to save them in data file with a given file name, as soon as the measurement is completed, as shown in Table $\mathrm{I}$.

The direct execution mode allows a user to modify the MCA parameters even when a measurement is running, and this is sometimes convenient for preliminary measurements or time-slicing experiments.

Table I shows an example of a VTL/K program along with the results of an execution. This program initializes the MCA by specifying the MCA parameters on line 130 (the line also activating the real time display), clears the data area on line 220 , starts the clock on line 230 and the data acquisition on 


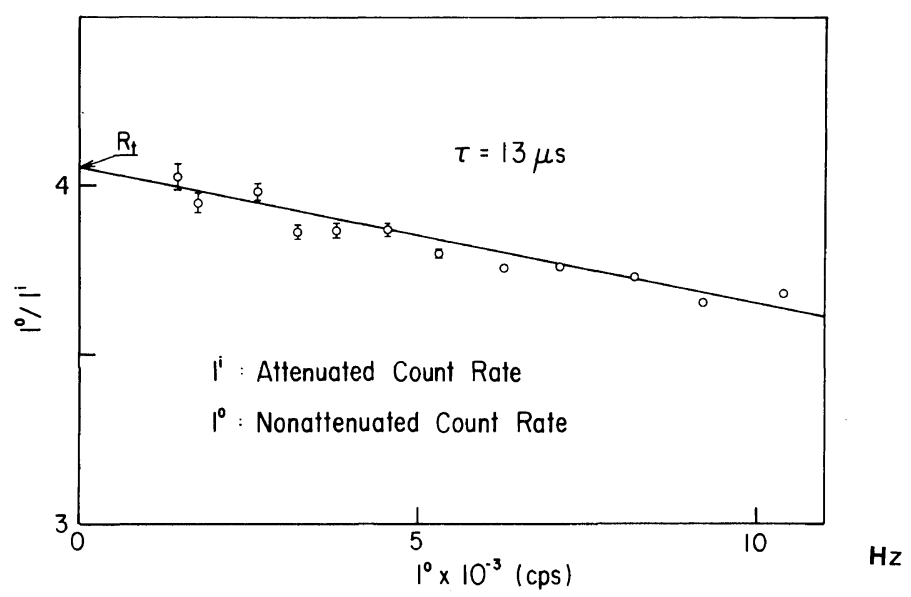

Figure 10. A plot for measuring the dead time of the PSD and associated electronics; $I^{0}$ is varied by changing the power of X-ray beam.

line 240. The subcommand "GO" on line 240 activates concurrently data acquisitions from the PSD with the MCA system and from the monitor proportional counter. After completion of the data acquisition, the program calculates the total intensity (in counts $\mathrm{s}^{-1}$ ) and the maximum intensity (in counts) and finds the channel of the maximum intensity (see the programs on the lines from 310 to 340). The program also prints out the intensity data (in counts) over 30 channels in the vicinity of the maximum intensity (see programs on the lines from 350 to 470). The results of such executions are shown in a form of a table (marked ** STATIC DATA $^{* *}$ ) in between the lines 5 and 9 from the bottom line of Table I. The 9 th line from the bottom indicates the total count (601 counts $\mathrm{s}^{-1}$ ), the maximum intensity (1423 counts), and the channel number showing the maximum intensity (28th ch.). The numbers 0 to 9 on the line immediately below this line specify the first order of the channel number, and the numbers 10 to 30 in the beginning of lines 7 to 5 from the bottom specify, respectively, the second order of the channel number. The remaining numbers in the "STATIC DATA" table show photon-counting data in counts.

The command, "\#=," followed by a line number executes a VTL/K program at the specified line number (see 13th line from the bottom). As soon as the execution is completed, the system comes to the supervisor mode, and is capable of accepting any commands including the system commands. The program includes a direct execution of the com- mand "EXP. TABLE" which prints the current MCA parameters as shown on the bottom two lines of the Table I.

The measured data are stored in cassette tape data file in a fixed format. A format conversion program is used to convert data in the file to cardimage files which can be sent to the University Computer Center through a telephone line.

\section{Some Performances on Detector}

Some performances of the PSD and its associated electronics are described in detail elsewhere. ${ }^{18}$ In this paper, we briefly summarize the results.

The uniformity of the detector sensitivity along its length was measured by $\mathrm{Fe} K \alpha$ radiation from $2 \mathrm{mCi}$ ${ }^{57} \mathrm{Co}$. The sensitivity is uniform within $\pm 3.5 \%$ and reproducible. The relationship between the channel number of the detector and position of photons was found to be linear within $\pm 2.4 \%$. The position resolution of the detector can be given by the Gaussian function, $R_{\mathrm{D}}(x)$ with a standard deviation $\sigma_{\mathrm{D}}$ of $59 \mu \mathrm{m}^{18}$ :

$R_{\mathrm{D}}(x)=\left(2 \pi \sigma_{\mathrm{D}}^{2}\right)^{-1 / 2} \exp \left(-x^{2} / 2 \sigma_{\mathrm{D}}^{2}\right), \quad \sigma_{\mathrm{D}}=59 \mu \mathrm{m}$

The resolution reduction of the detector due to an oblique incidence of photons depends only on the angle of incidence and the depth of the detector along the $z$-direction, and the associated smoothing function is given purely by from a geometrical relationship. ${ }^{18}$ The quantum efficiency of the detector for $\mathrm{Cu} K \alpha$ radiation is $38 \%$ for Argon-gas flow $\left(\mathrm{Ar} 90 \%\right.$ and $\mathrm{CH}_{4} 10 \%$ ) at $3.0 \mathrm{~atm}$. pressure. 
The energy resolution of the detector was determined from a differential curve on energy distribution. The energy resolution thus measured was $18 \%$ for typical SAXS intensity levels used in our measurements.

The dead time of the PSD itself was $800 \mathrm{~ns}$. The dead time of the system as a whole was determined according to Chipman's method. ${ }^{19}$ We measured the nominal counting rates $I^{\mathrm{i}}$ and $I^{0}$ of scattered radiation with and without $\mathrm{Ni}$ filter in the beam path, respectively. The ratio $R \equiv I^{0} / I^{\mathrm{i}}$ is related to the true ratio $R_{\mathrm{t}}$ (which may be obtained in case when there is no counting loss) and $\tau$, the dead time of the systems as,

$$
R=R_{\mathrm{t}}-I^{0} \tau\left(R_{\mathrm{t}}-1\right)
$$

Thus, $\tau$ is obtained from the intercept (at $I^{0}=0$ ) and the slope in the plot of $R v s . I^{0}$ (as shown in Figure $10), I^{0}$ being varied by changing the power of $\mathrm{X}$-ray beam. The dead time thus estimated was $13 \pm 3 \mu \mathrm{s}$; this value may be reasonable in the light of the designed MCA function as described in the previous section (3).*

\section{SOME APPLICATIONS OF DYNAMIC SAXS MEASUREMENTS TO POLYMERIC SYSTEMS}

\section{Dynamics in Phase-Separation and Phase-} Annihilation in Multicomponent Polymer Systems As an example of multicomponent polymer systems, we consider here polystyrene and polyisoprene block polymers (SI). Due to the repulsive interaction between the constituent polymers, the block polymers undergo microphase separation, resulting in a.microdomain structure in the solid state. ${ }^{20,21}$ Since the mixture of polystyrene and polyisoprene exhibit the upper critical solution tem-

* The data acquisition system is designed in such a way that the net dead time of the system is determined by the dead time of either the ADC or the memory incrementing cycle, the step having longer dead time being the ratedetermining step of the entire system. Since the memory incrementing cycle is measured to be $11 \mu \mathrm{s}$, the measured dead time of $13 \mu \mathrm{s}$ of the entire system should reflect the dead time of the ADC. The measured dead time of $13 \mu \mathrm{s}$ is rationalized because the net dead time for the ADC is a sum of the time required for the conversion $(10 \mu \mathrm{s})$, the time for the acceptance test $(1 \mu \mathrm{s})$, and the hold time (set to $2 \mu \mathrm{s})$.

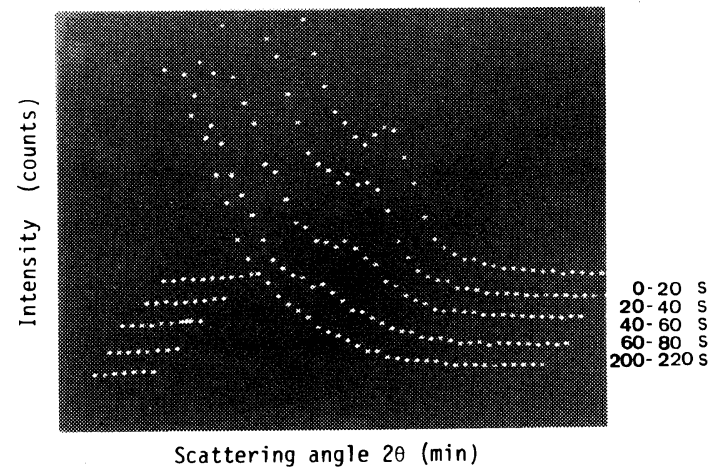

Figure 11. Typical change in SAXS profiles with time from a SI block polymer after a temperature- jump from room temperature to $200^{\circ} \mathrm{C}$, which is above the critical temperature $\left(170^{\circ} \mathrm{C}\right)$.

perature (UCST) $)^{22}$ so that the microdomain structure melts to a homogeneous mixture on elevating the temperature above the critical temperature $T_{\mathrm{c}}^{16,23,24}$ (a "phase-annihilation" process).

Figure 11 represents a typical change in the SAXS profiles for a particular SI block polymer, (more rigorously, a "tapered" SI block polymer ${ }^{25}$ having a critical temperature $T_{\mathrm{c}} \simeq 170^{\circ} \mathrm{C}$ ) with time after a temperature-jump from room temperature to $200^{\circ} \mathrm{C}$. The experiment was conducted by setting the MCA parameters as described in the previous section 4 as follows; $\mathrm{PT}=20, \mathrm{TI}=0, \mathrm{FS}=4, \mathrm{XC}=256, \mathrm{YC}$ $=24$, and so on. Therefore, each curve was measured with an exposure time (PT) of $20 \mathrm{sec}$ and one channel corresponded to $1 \mathrm{~min}$ in scattering angle. In the oscilloscope displays, the origin of each scattering curve is shifted diagonally to avoid overlap. The first several channels in each curve are covered by the beam stopper so that their photon-counts are extremely small. The SI block had a total molecular weight of $M_{n}=4.3 \times 10^{4}$, heterogeneity index $M_{w} / M_{n}=1.05$, and weight fraction of styrene $0.47 .^{25}$

It should be noted that the scattering maximum corresponding to the long identity period of the microdomain structure in the block polymer disappears with time in a period as short as $200 \mathrm{~s}$. The rate of intensity decay depends on the translational diffusion coefficient of the block polymer molecule in bulk accompanied by the phase transition. ${ }^{16,17}$

Figure 12 represents the changes in the SAXS curve with time after a temperature-jump from 
SBS-53 wt \% Dipentene Soln.

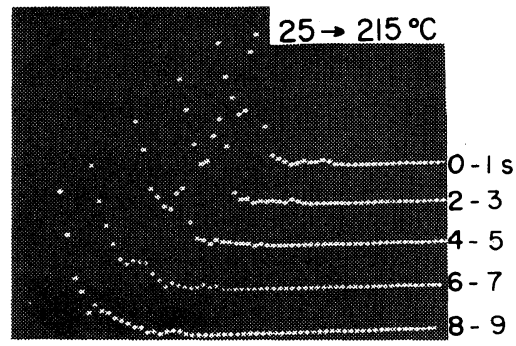

(a) Phase annihilation

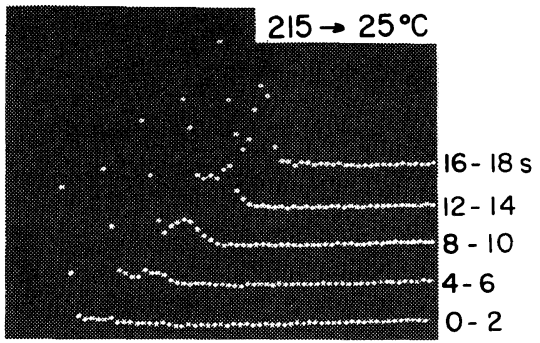

(b) Phase separation

Figure 12. A typical change in SAXS curves with time after a temperature-jump from room temperature to $215^{\circ} \mathrm{C}$ (a) (annihilation or melting process) and a drop from $215^{\circ} \mathrm{C}$ to room temperature (b) (process of phaseparation). The specimen was a $53 \mathrm{wt} \%$ dipentene solution of SBS triblock polymer having a critical temperature of $150^{\circ} \mathrm{C}$.

room temperature to $215^{\circ} \mathrm{C}$ (a) and a drop from $215^{\circ} \mathrm{C}$ to room temperature (b). The specimen studied was a $53 \mathrm{wt} \%$ dipentene solution of polystyrene-polybutadiene-polystyrene triblock polymer (SBS). The block polymer had molecular weights of 14-30-14 for each block sequence in units of thousands. Even in solution, the block polymers form the microdomain structure at concentrations above the critical micelle concentrations. ${ }^{20,21,26,27}$ The scattering peak corresponds to the long identity period of the microdomain structure in solution.

Figures 12(a) and 12(b) correspond, respectively, to a process in which the phase-separated structure becomes a homogeneous mixture and a process in which the phase-separated structure is formed from a homogeneous mixture ( $T_{\mathrm{c}}$ for this system being $150^{\circ} \mathrm{C}$ ). In Figures 12(a) and 12(b), each curve was obtained with a measuring time (PT) of $1 \mathrm{~s}$ and $2 \mathrm{~s}$, respectively.

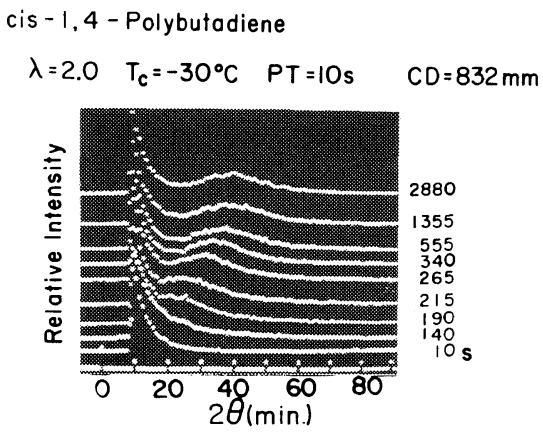

(a)

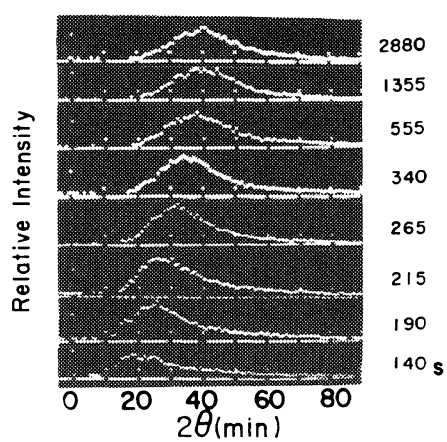

(b)

Figure 13. (a) A typical change in SAXS curves with time following isothermal crystallization of the stretched and crosslinked polybutadiene; the draw ratio of the specimen and crystallization temperature are 2.0 and $-30^{\circ} \mathrm{C}$, respectively. (b) The same data as in 13(a) except that each curve in Figure 13(b) was obtained after subtracting the scattered intensity of the stretched molten specimen from the scattered intensity shown in Figure 13(a).

This study can be easily extended to polymer blends in which can be examined the formation of the structure through a process involving nucleation and growth or a process involving spinodal decomposition $^{28-30}$ as well as the annihilation of the phase-separated structure. ${ }^{17}$

\section{Kinetics of Crystallization}

The dynamic SAXS technique can be applied to studying the kinetics of polymer crystallization. The time-resolved SAXS measurements involve analyses of the radius of gyration, invariant, and long identity period as a function of time during isothermal crystallization, providing fundamental information on nucleation and growth of polymer crystals.

Figure 13 represents a typical change in SAXS 
curves with time following the isothermal crystallization of stretched and crosslinked polybutadiene, with the draw ratio of the specimen and the crystallization temperature being 2.0 and $-30^{\circ} \mathrm{C}$, respectively. The details of these specimens are described elsewhere. ${ }^{38}$ The measuring time for each SAXS curve was $10 \mathrm{~s}$ and each curve was shifted vertically to avoid overlap. In Figure 13(a), the beam-stopper covers the angular region up to 10 minute in the scattering angle.

The SAXS pattern taken with a Huxley-Holmes camera (an optics composed of a bent mirror and a bent-crystal monochromator) shows a typical twopoint pattern which appeared parallel to the draw direction. Figure 13(a) shows the SAXS profiles measured with the PSD along the draw direction. It is clear that the scattering intensity increases with time, i.e., as crystallization proceeds, and that a scattering maximum due to a long identity period appears. The intensity maximum increases and shifts toward a large scattering angle with time. The decrease in the long identity period with time may be interpreted as a decrease in crystal thickness and/or a decrease in the intercrystal amorphous zone along the draw direction; verification of this will require further investigations. Figure 13(b) shows the same data as in Figure 13(a) but each curve in Figure 13(b) was obtained after subtracting the scattering intensity for stretched molten specimen from each scattering intensity shown in Figure 13(a), the calculation being conducted with the $\mathrm{VTL} / \mathrm{K}$ in a manner as described in detail in the previous section 4 . The increase in the peak intensity and the shift of the peak position with time can be seen more clearly in Figure 13(b) than in Figure 13(a).

\section{Structure Change in Ion-Containing Polymer}

As a third example of applying the dynamic SAXS technique to a polymer system, Figure 14 represents the change in SAXS curves with time after a film specimen of an ion-containing polymer immersed in water at room temperature. The polymer studied here is "Nafion," registered trademark of E.I. du Pont de Nemours and Company, Inc. for its perfluorosulfonic acid products. This polymer consists of a tetrafluoroethylene backbone, with a perfluoronated ether side chains terminated by an $\mathrm{SO}_{3} \mathrm{H}$ groups (Nafion-H). The E.W. (equivalent weight $)^{31}$ of the specimen studied here was 1100 .

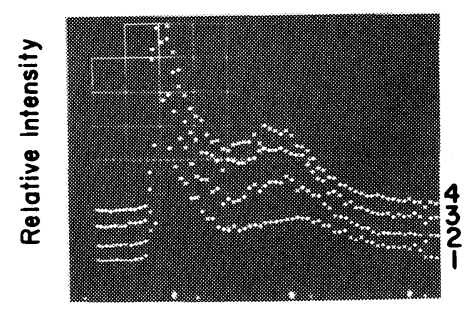

Scattering Angle

(a)

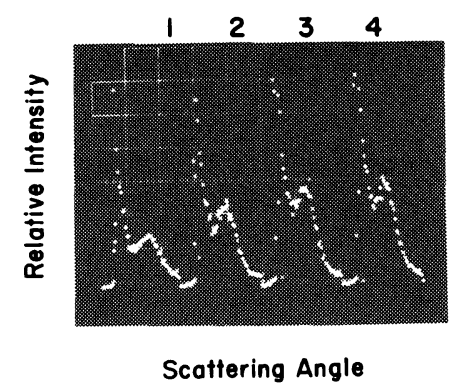

(b)

Nafion $\left(\mathrm{SO}_{3} \mathrm{H}\right)-\mathrm{H}_{2} \mathrm{O}$

$1,0-10 \mathrm{~s} ; 2,10-20 \mathrm{~s} ; 3,20-30 \mathrm{~s} ; 4,30-40 \mathrm{~s}$.

Figure 14. Change in SAXS curves with time after the film specimen of the ion-containing polymer (Nafion-H) was immersed in water at room temperature. Curves 1 to 4 were obtained in time intervals of 0 to 10,10 to 20,20 to 30 , and 30 to 40 seconds after the sample was quickly immersed in water.

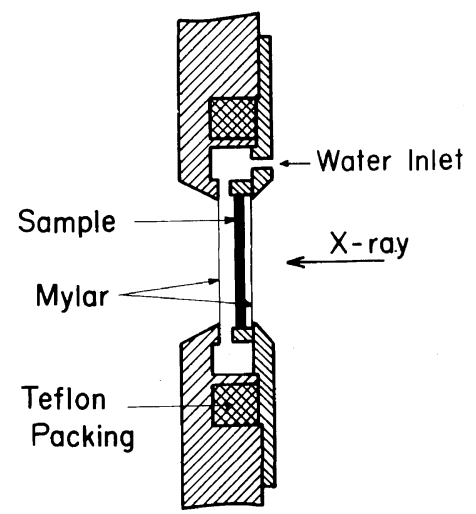

Figure 15. A sample cell used in the time-dependent swelling experiments shown in Figure 14.

It has been proposed that the ionic groups in the polymer form ionic clusters. ${ }^{31-33}$ The SAXS peak shown in Figure 14 is attributed to interparticles $^{32,33}$ or the intraparticle interference ${ }^{33}$ of the 


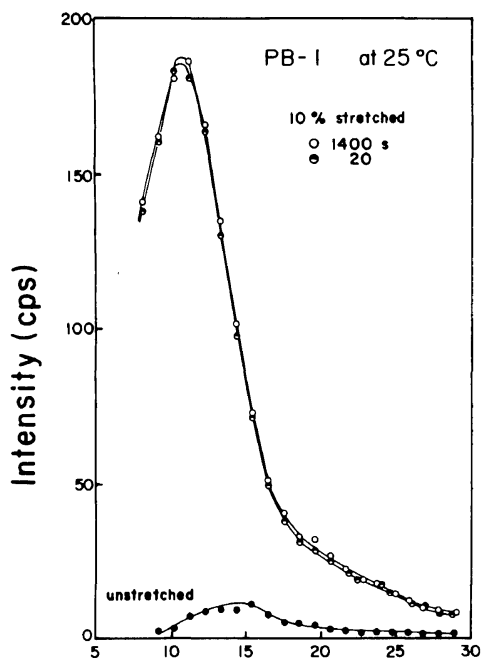

(a)

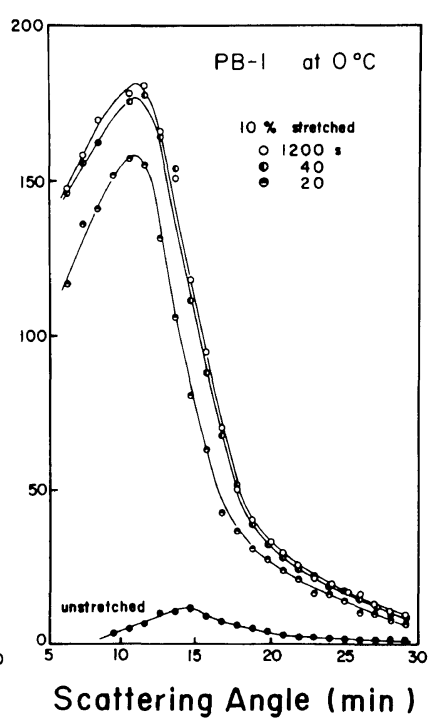

(b)

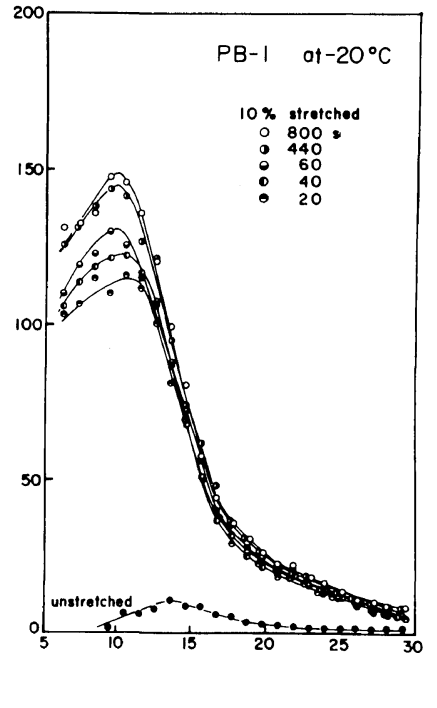

(c)

Figure 16. Change in SAXS curves with time during the course of the stress relaxation of the blown poly(1-butene) film. Each curve was obtained by a $20 \mathrm{~s}$ exposure to X-ray at times specified in the figure following a rapid stretching of specimens by $10 \%$ along the machine direcion.

ionic clusters. Figure 14 shows the change in ionic clusters on immersing the specimens in water. Figure 15 illustrates schematically the sample cell used in the experiment. The film specimen was clamped at one end in the cell. Immediately after the cell is filled with water, the dynamic experiment is started. The curves numbered 1 to 4 in Figure 14 are taken consecutively with a measuring time of $10 \mathrm{~s}$ $(\mathrm{PT}=10, \mathrm{TI}=0)$. In Figure 14(a) the origin of each curve is shifted vertically to facilitate the investigation of the change in peak shifts, while in Figure 14(b) the origin is shifted horizontally to facilitate the investigation of change in peak intensity. It is seen that as the specimen becomes swollen with water, the SAXS peak shifts to smaller angles (from 174 to 116 minutes in the scattering angle, $\mathrm{Cu} \mathrm{K \alpha}$ radiation), suggesting the growth of ionic clusters. The peak intensity increases due to increased cluster size and greater contrast between the clusters and surrounding medium. The intensity change with time should reflect the diffusion coefficient of water in the film.

\section{Dynamics in Deformation of Crystalline Polymers}

The technique can be applied to the study of the dynamics of polymer deformation which involves the initiation and growth of crazes and cracks in glassy polymers, and the deformation and orientation of lamellar platelets and interlamellar amorphous chains in crystalline polymers.

Figure 16 represents the change in SAXS curves with time during the course of the stress relaxation of blown poly(1-butene) (PB-1) films. Each curve was obtained with $20 \mathrm{~s}$ exposure to X-ray at times specified in the figure following rapid stretching.

The specimens have row-nucleated structures containing oriented lamellar plates whose chain axes run parallel to the machine direction (MD). The lamellae are stacked regularly along the MD with well defined long identity periods. ${ }^{34-37}$ The specimens rapidly stretched by $10 \%$ along the machine direction at 25,0 , and $-20^{\circ} \mathrm{C}$.

On stretching the film along the MD, the interlamellar spacing increases, causing a lowering of the density of the interlamellar amorphous region, resulting in an increase in the density difference between the crystal lamellae and amorphous region. ${ }^{36,37}$ This type of deformation involves a tremendous increase in the scattered intensity and a shift of the scattering maximum toward smaller scattering angles, ${ }^{34,36}$ as shown in Figure 16.

The deformation at room temperature takes place 
instantaneously as observed in the instantaneous change in the SAXS curves; this change is completed within $20 \mathrm{~s}$. However with a decrease in the temperature, the deformation becomes timedependent with greater relaxation time as shown in Figures 16(b), and 16(c). The time-dependence of SAXS profiles and intensities are primarily related to the mobility of interlamellar amorphous materials, i.e., the relaxation time for amorphous chains to orient and/or deform. Deformation of the interlamellar spacing involves the deformation and orientation of amorphous chains in the interlamellar region. The deformation and orientation of the amorphous chains occur instantaneously when stress is applied at room temperature which is well above the glass transition temperature $\left(T_{\mathrm{g}}\right)$ of PB-1. As the temperature is lowered toward the $T_{\mathrm{g}}$, the relaxation time for the deformation and orientation of the amorphous material increases, and consequently, a viscous drag of the amorphous phase on the deformation of interlamellar spacing increases, resulting in greater relaxation time for the change in SAXS intensity and profile. The relaxation time is obviously affected strongly by the nature of the amorphous phase in the crystalline polymers.

\section{CONCLUDING REMARKS}

It is obvious in the light of the preliminary experimental results shown in this paper that one can follow the time-dependent structural changes of a few hundred Angstroms by the SAXS technique. The dynamic SAXS technique undoubtedly provides a new source of experimental evidence to that obtained by other techniques, including rheooptical techniques employed in the study of the structure-property relationships in polymeric systems.

Acknowledgements. This work was supported by a Grant-in-Aid for Scientific Research (243021 and 449012) from the Ministry of Education, Science and Culture Japan. We should like to thank the Rigaku Denki Corporation, Tokyo, Japan, and Mr. K. Sugimoto, HASC Engineering Corporation, Kyoto, Japan, for technical support.

\section{REFERENCES}

1. Y. Dupont, M. Gabriel, M. Charbre, T. GulikKrezywicki, and E. Schelter, Nature (London), 238, 332 (1972).

2. J. Schelten and R. W. Hendricks, J. Appl. Crystallogr., 8, 421 (1975).

3. H. Hashizume, H. Nakajima, K. Inoda, Y. Amemiya, M. Itoh, T. Nishijima, and K. Kohra, Jpn. J. Appl. Phys., 15, 2211 (1976).

4. R. J. Podolsky, R. St. Onge, L. Yu, and R. W. Lymn, Proc. Nat. Acad. Sci., 73, 813 (1976).

5. A. R. Faruqi and H. E. Huxley, J. Appl. Crystallogr., 11, 449 (1978).

6. C. J. Borkowski and M. K. Kopp, IEEE Trans. Nucl. Sci., NS-17, 340 (1970).

7. C. J. Borkowski and M. K. Kopp, IEEE Trans. Nucl. Sci., NS-19, 161 (1972).

8. C. Cork, D. Fehr, R. Hamlin, W. Vernon, V. PerezMendez, and Ng. H. Xuong, J. Appl. Crystallogr., 7, 319 (1974).

9. R. W. Hendricks, J. Appl. Crystallogr., 11, 15 (1978).

10. R. W. Hendricks, Trans. Am. Crystallogr. Assoc., 12, 103 (1976).

11. G. Rosenbaum, K. C. Holmes, and J. Witz, Nature (London), 230, 434 (1971).

12. J. Barrington Leigh and G. Rosenbaum, J. Appl. Crystallogr., 7, 117 (1974).

13. J. C. Phillips, A. Wlodawer, M. M. Yevitz, and K. O. Hodgson, Proc. Natl. Acad. Sci., 73, $128-132$ (1976).

14. K. O. Hodgson, Trans. ACA, 12, 1 (1976).

15. (a) J. M. Schultz, J. S. Lin, and R. W. Hendricks, J. Appl. Crystallogr., 11, 551 (1978).

(b) K: Matsushige, K. Nagata, and T. Takemura, Jpn. J. Appl. Phys., 17, 467 (1978).

16. T. Hashimoto, Y. Tsukahara, and H. Kawai, $J$. polym. Sci., Polym. Lett. Ed., 18, 585 (1980).

17. T. Hashimoto, Y. Tsukahara, and H. Kawai, Macromolecules, 13, 000 (1981).

18. M. Fujimura, T. Hashimoto, and H. Kawai, Mem. Fac. Eng., Kyoto Univ., (1981), 43(2) in press.

19. D. R. Chipman, Acta Cryst., A25, 209 (1969).

20. G. E. Molau, in "Block Polymers," S. L. Aggarwal, Ed., Plenum, N.Y. (1970).

21. T. Inoue, T. Soen, T. Hashimoto, and H. Kawai, $J$. Polym. Sci., A-2, 7, 1283 (1969).

22. R. S. Scott, J. Chem. Phys., 17, 279 (1949).

23. D. McIntyre, N. Rounds, and E. Campos-Lopez Am. Chem. Soc., Polym., Prepr., 10, 531 (1969).

24. R. Koningsveld, L. A. Kleintjens, and H. M. Schoffelecrs, Pure Appl. Chem., 39, 1 (1974).

25. Y. Tsukahara, N. Nakamura, T. Hashimoto, H. Kawai, T. Nagaya, Y. Sugimura, and S. Tsuge, Polym. J., 12, 455 (1980).

26. C. Sadron and B. Gallot, Makromol. Chem., 164, 301 (1973). 
27. T. Hashimoto, M. Shibayama, M. Fujimura, and H. Kawai, in "Block Copolymers: Science and Technology," D. J. Meier, Ed., MMI press, Gordon \& Breach Science Midland, Michigan, in press; Mem. Fac. Eng., Kyoto Univ., (1981), 43(2) in press.

28. J. W. Cahn, Acta Metall., 9, 795 (1961).

29. J. W. Cahn, Acta Metall., 10, 907 (1962).

30. J. J. van Aartsen, Eur. Polyrn. J., 6, 919 (1970).

31. S. C. Yeo and A. Eisenberg, J. Appl. Polym., Sci., 21, 875 (1977).

32. B. Rodmacq, J. M. Coey, M. Escoubez, E. Roche, R. Duplessix, A. Eisenberg, and M. Pineri, Polym. Prepr., J. Am. Chem. Soc., Div. Polym. Chem., 20, 670 (1979).

33. M. Fujimura, T. Hashimoto, and H. Kawai, submitted to Macromolecules.

34. T. Hashimoto, A. Todo, Y. Murakami, and H.
Kawai, J. Polym. Sci., Polym. Phys. Ed., 15, 501 (1977).

35. T. Hashimoto, A. Todo, and H. Kawai, Polym. J., 10, 521 (1978).

36. T. Hashimoto, A. Todo, Y. Tsukahara, and $\mathrm{H}$. Kawai, Polymer, 20, 636 (1979).

37. A. Todo, T. Hashimoto, Y. Tsukahara, and $\mathrm{H}$. Kawai, Polymer, 20, 943 (1979).

38. A. Wasiak, K. Saijo, M. Fujimura, T. Hashimoto, and $\mathrm{H}$. Kawai, to be published.

39. R. W. Hendricks, P. A. Seeger, J. W. Scheer, and S. Suehiro, Oak Ridge National Laboratory Report ORNL/TM-7325 (1980); Nucl. Instr. Methods, in press.

40. S. Suehiro, Interface (Jpn), 4(2), 83 (1978).

41. MITS Inc.,A Very Tiny Language for the Altair 680 (1976). 